\title{
Neuroretina specification in mouse embryos requires Six3-mediated suppression of Wnt8b in the anterior neural plate
}

\author{
Wei Liu, ${ }^{1}$ Oleg Lagutin, ${ }^{1}$ Eric Swindell, ${ }^{2}$ Milan Jamrich, ${ }^{3}$ and Guillermo Oliver $^{1}$ \\ 1Department of Genetics and Tumor Cell Biology, St. Jude Children's Research Hospital, Memphis, Tennessee, USA. \\ 2Department of Pediatrics, The University of Texas Medical School at Houston, Houston, Texas, USA. \\ ${ }^{3}$ Department of Molecular and Cellular Biology, Baylor College of Medicine, Houston, Texas, USA.
}

\begin{abstract}
Retinal degeneration causes vision impairment and blindness in humans. If one day we are to harness the potential of stem cell-based cell replacement therapies to treat these conditions, it is imperative that we better understand normal retina development. Currently, the genes and mechanisms that regulate the specification of the neuroretina during vertebrate eye development remain unknown. Here, we identify sine oculis-related homeobox 3 (Six3) as a crucial player in this process in mice. In Six 3 conditional-mutant mouse embryos, specification of the neuroretina was abrogated, but that of the retinal pigmented epithelium was normal. Conditional deletion of Six 3 did not affect the initial development of the optic vesicle but did arrest subsequent neuroretina specification. Ectopic rostral expansion of Wnt8b expression was the major response to Six 3 deletion and the leading cause for the specific lack of neuroretina, as ectopic Wnt8b expression in transgenic embryos was sufficient to suppress neuroretina specification. Using chromatin immunoprecipitation assays, we identified Six3-responsive elements in the Wnt8b locus and demonstrated that Six 3 directly repressed Wnt8b expression in vivo. Our findings provide a molecular framework to the program leading to neuroretina differentiation and may be relevant for the development of novel strategies aimed at characterizing and eventually treating different abnormalities in eye formation.
\end{abstract}

\section{Introduction}

Various human ocular diseases due to retinal degeneration lead to vision impairment and eventually blindness. Recent advances using embryonic stem cells and induced pluripotent stem cells suggest that one day cell-replacement therapy will be used to treat some of these diseases $(1,2)$. A prerequisite to achieving this goal is to better understand the regulatory cascade and signaling pathways that control normal retina development.

In vertebrates, the retina is composed of the neuroretina (NR) and the retina pigment epithelium (RPE), both derived from common progenitors located in the early eye field and its derivative, the optic vesicles (3). In the mouse, fate-mapping analysis of 5- to 7-somite staged (i.e., E8.5) embryos places the eye field in the rostral anterior neural plate (4). As a result of coordinated interactions of the neuroepithelium, overlying surface ectoderm, and mesenchyme, the eye field undergoes extensive morphogenesis to form the optic vesicles (E9.0-E9.5) and optic cup (E10.5). At E9.5, the optic vesicle and the overlying surface ectoderm are in close contact. At E10.5, the double-layered optic cups form, and the NR and RPE become morphologically distinguishable, i.e., NR progenitors are localized in the inner layer and RPE progenitors are in the outer layer. Eventually, NR progenitors give rise to the 6 major types of differentiated neurons and 1 type of glial cell in the mature neuroretina (5).

Similar to the eye field of the frog $(6,7)$, that of the mouse expresses a series of characteristic transcription factors including Six3, Rax (also known as Rx), Lhx2, and Pax6 (8). Later, during the formation of the optic vesicles (E9.0-E9.5), additional genes are

Conflict of interest: The authors have declared that no conflict of interest exists. Citation for this article: J Clin Invest. 2010;120(10):3568-3577. doi:10.1172/JCI43219. expressed, including Six6 in presumptive NR progenitors (9), Vax2 in the ventral optic vesicles and optic stalk (10), Tbx5 in the dorsal optic vesicles (11), and the bHLH leucine-zipper transcription factor Mitf in the whole optic vesicle (12). At around E10.5, nascent NR progenitors are marked by the onset of $V s \times 2$ (also known as Chx10) expression, and the RPE progenitors are marked by the restricted expression of $\operatorname{Mitf}(12)$.

In the mouse, some key genes responsible for eye development have been identified. For example, formation of the eye field and optic vesicles requires the activity of $L h x 2$ and $\operatorname{Rax}(8,13-16)$. Functional inactivation of Pax 6 affects the formation of the optic cup; however, NR specification is normal, as indicated by the expression of the NR progenitor marker $V s x 2$ (17). In contrast to the crucial role of $F z d 5$ in zebrafish eye development, functional inactivation of $F z d 5$ in mice causes defects only in the invagination of the optic vesicles; NR specification is not affected (18). Functional inactivation of Six6 (19), $\operatorname{Vax} 2(10), \operatorname{Vsx} 2(20,21)$, or Mitf(22) causes some later defects in eye development but does not affect NR specification.

Despite this progress, the molecular mechanisms controlling the key step of NR specification during vertebrate eye development remain largely unknown.

The homeobox gene Six 3 has a dynamic expression profile during early forebrain and eye development (23), and previous work has determined that Six 3 activity is essential for forebrain formation (24). In this paper, we show that conditional removal of Six3 from the developing mouse eye field is sufficient to arrest NR specification. In the generated Six 3 conditional mutant embryos, eye field induction was not affected; however, NR specification did not take place in the mutant optic vesicles, but RPE specification appeared normal. Therefore, this is the first animal model in which NR specification is specifically arrested. We provide evi- 

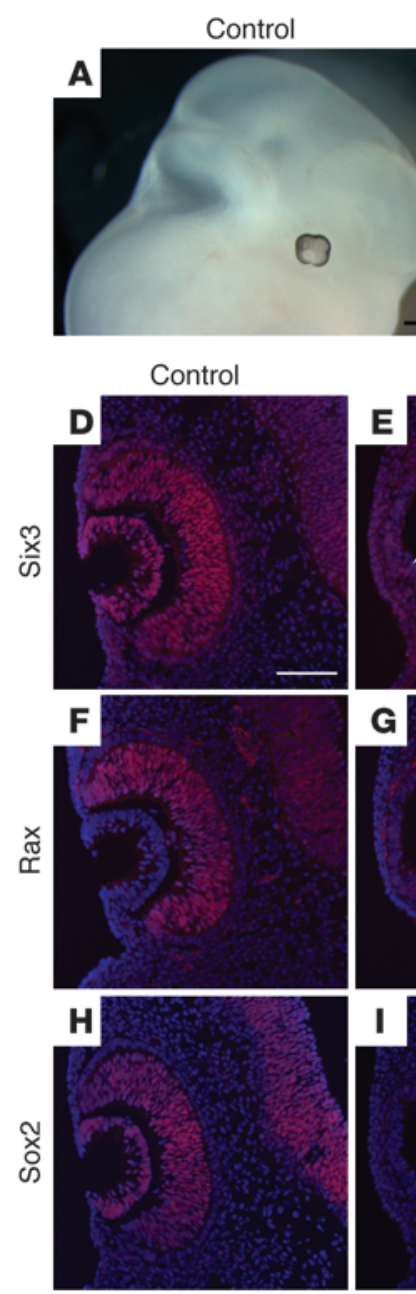

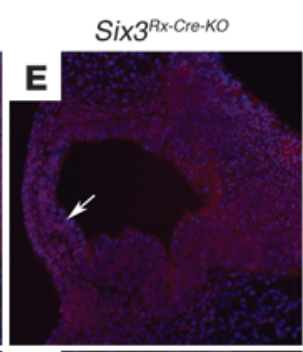

G
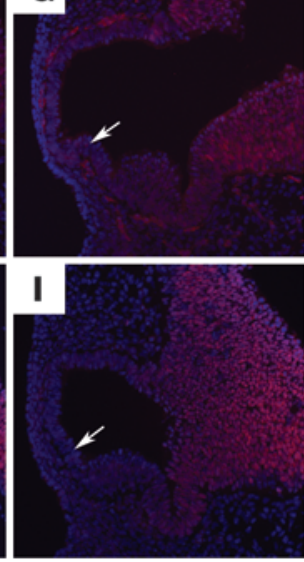

Six $3^{\text {Rx-Cre-KO }}$

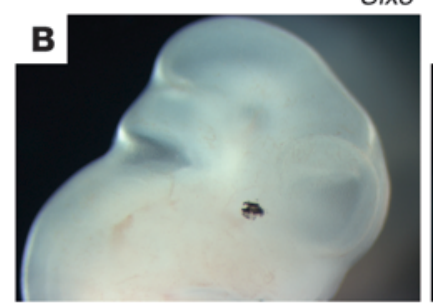

Control
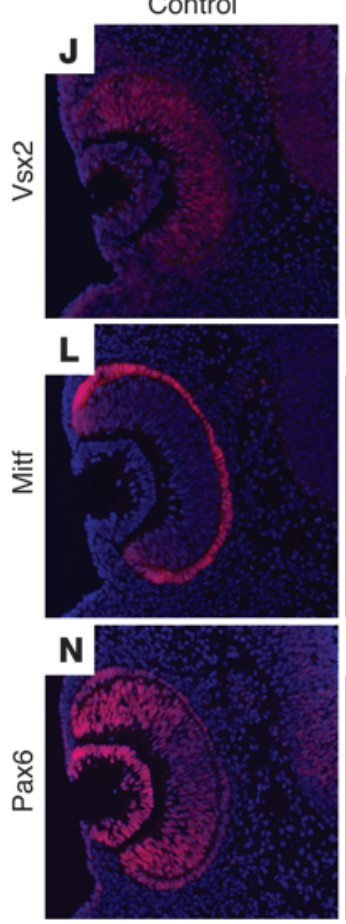

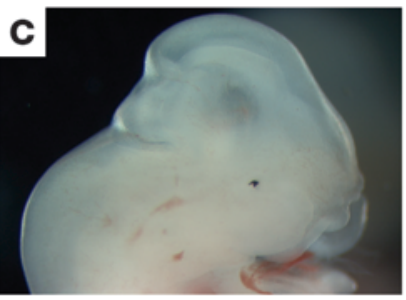

$\operatorname{Six}^{\mathrm{Rx} \cdot \mathrm{Cre}-\mathrm{KO}}$

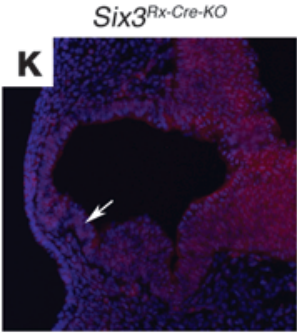

M
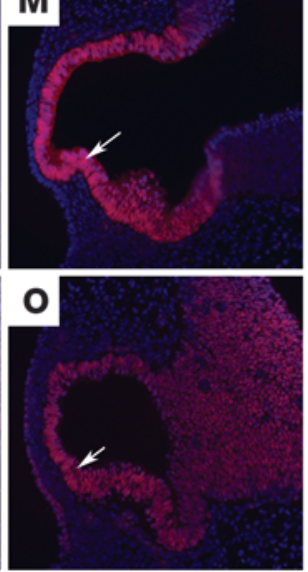

\section{Figure 1}

Six 3 activity is crucial for the development of the mammalian neuroretina. (A) At E11.5, a normal-looking pigmented eye is seen in control embryos. In Six3 conditional-mutant littermates, the eye appears defective and composed of only the RPE (B and C). At E10.5, NR specification has already taken place in control embryos, as indicated by the expression of the NR markers Six3 (D), Rax (F), Sox2 (H), and $\operatorname{Vsx} 2(\mathbf{J})$. In the conditionalmutant littermates, this process is defective, as revealed by the lack of expression of any of these NR markers (arrows in $\mathbf{E}, \mathbf{G}, \mathbf{I}$ and $\mathbf{K}$ ). At this stage, the RPE is also normally detected, as indicated by the expression of Mitf (L) and Pax6 (N). In Six3 conditional-mutant embryos, the expression of these 2 transcription factors highlights the fact that the mutant optic vesicle is exclusively composed of the RPE (arrows in $\mathbf{M}$ and O). Scale bars: $500 \mu \mathrm{m}(\mathbf{A}-\mathbf{C})$; $100 \mu \mathrm{m}$ (D-0). dence that Six3-mediated direct suppression of $W n t 8 b$ is a required step for NR specification in mammals and that a tightly regulated temporal and spatial balance of Wnt/ $\beta$-catenin signaling is essential for telencephalon, NR, and RPE specification.

\section{Results}

Six 3 conditional-mutant embryos lack the NR but exhibit a normal RPE. To address the role of Six 3 in NR development, we generated Six $3^{F / \Delta}$; $R x$-Cre mutant embryos (referred to as Six $3^{R x-C r e-K O}$ hereafter) by crossing the floxed Six 3 mouse strain (25) with the $R x$-Cre strain (26). Activity of $R x$-Cre was associated with retina-forming cells located in the anterior neural plate and eye field at around E8.5 (Supplemental Figure 1; supplemental material available online with this article; doi:10.1172/JCI43219DS1) (26). Initial morphologic analysis of Six3 conditional-mutant embryos at E11.5 revealed that most exhibited a relatively normal-looking brain, though the brain was reduced in size in some embryos. The eyes of these embryos also appeared smaller and composed exclusively of pigmented cells (Figure 1, B and C). A low percentage of the isolated mutant embryos lacked a forebrain, a phenotype similar to that previously reported for the standard Six3-null embryos (24).

Next we performed a detailed characterization of conditionalmutant embryos at E10.5, a stage when the NR and RPE are easily distinguished by the use of molecular markers. This analysis revealed the presence of a mutant optic cup devoid of Six3 expression (Figure 1E). Other typical NR markers such as Rax, Sox2, and Vsx2 were also missing (Figure 1, G, I, and K). In contrast, the entire mutant optic vesicle expressed the RPE-specific marker Mitf (Figure 1M) as well as Pax6, a gene that at this stage is normally expressed by both the NR and RPE (Figure 1, N and O).

Together, these results identified Six 3 as a gene whose activity is required for the development of the mammalian NR but not for the formation of the RPE.

$N R$ specification is arrested in Six $3^{R x-C r e-K O}$ embryos. To pinpoint the developmental phase when phenotypic alterations arise, we performed a molecular characterization of the mutant embryos at the optic vesicle stage (E9.0-E9.5).

The frizzled family member $F z d 5$ is expressed in the eye field, developing optic cups, and retina, and in frogs, its activity is required to promote the neural fate of retinal progenitors (18, 27-30). Unlike that in frogs, $F z d 5$ activity in mammals is required for optic cup morphogenesis but not for NR specification (18). Strong Fzd5 expression was detected in the anterior part of the ventral forebrain and optic vesicles of E9.0 control embryos (Figure 2A); in contrast, it was absent in Six $3^{R x-C r e-K O}$ littermates (Figure 2B).

The homeobox-containing gene Rax is required for the formation of the optic vesicles and all of its derivatives in vertebrate embryos $(14,30)$. At the 15 -somite stage, Rax expression was local- 

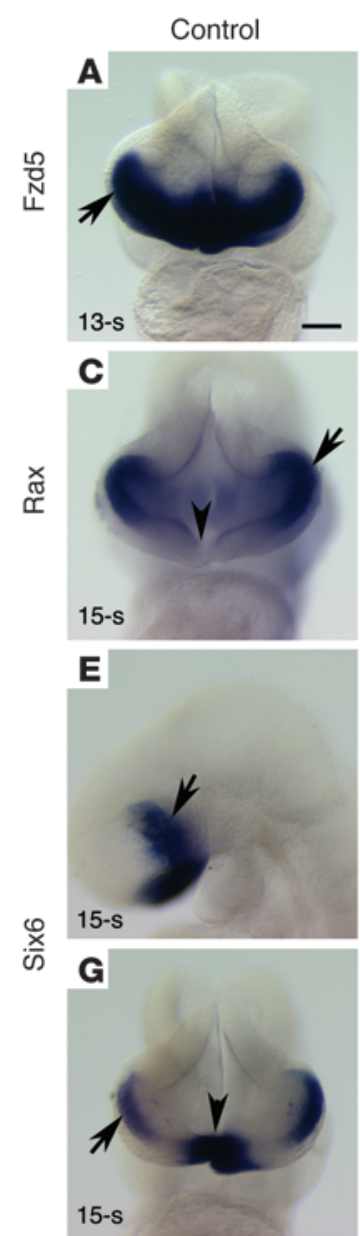
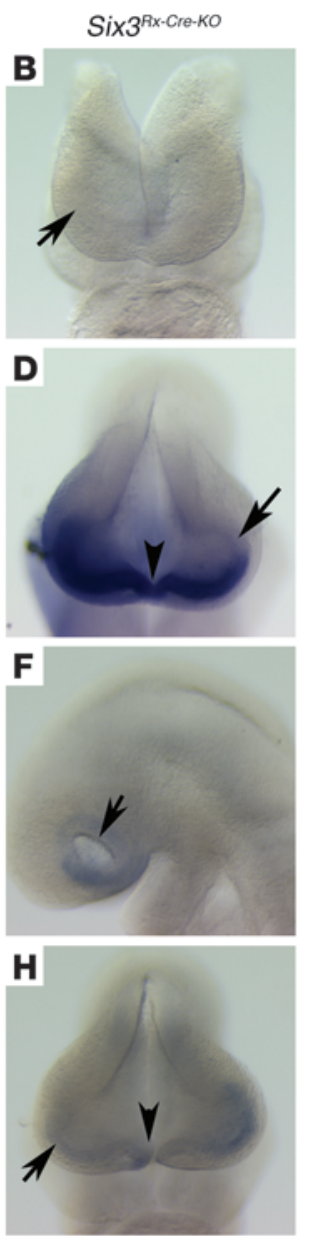
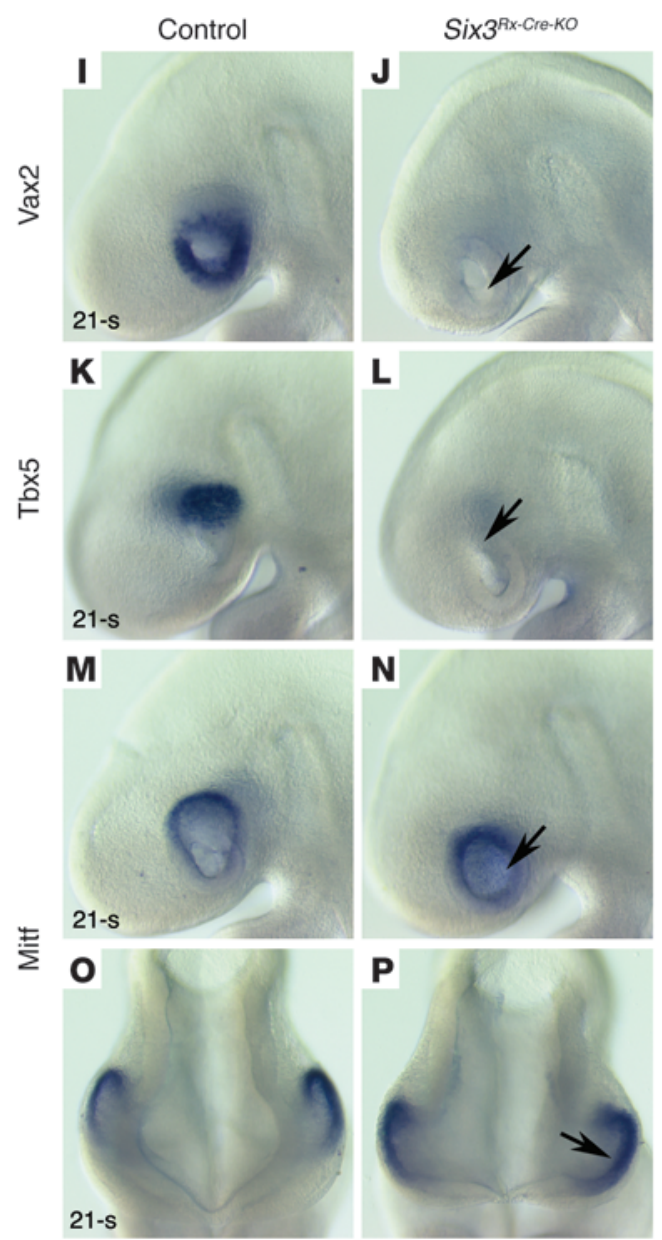

\section{Figure 2}

Expression of typical optic vesicle markers is missing or defective in Six3 conditional-mutant embryos. (A) At the 13-somite stage, Fzd5 expression is normally seen in the optic vesicles (arrow) and ventral forebrain of control embryos. (B) This expression was absent in the Six3-mutant littermates. (C) At the 15-somite stage, normal Rax expression is seen in the optic vesicles (arrow). (D) In the mutant littermates, Rax expression is missing from the dorsal optic vesicles and does not separate properly in the ventral midline region of the forebrain (arrowhead). (E and $\mathbf{G})$ At this same stage, Six6 expression is seen in presumptive NR progenitors in the optic vesicles (arrows) and ventral forebrain (arrowhead). ( $\mathbf{F}$ and $\mathbf{H})$ In Six3 conditional-mutant littermates, Six6 expression was almost completely absent from those territories (arrow and arrowhead). At the 21-somite stage, expression of the ventral (Vax2; I and $\mathbf{J}$ ) and dorsal (Tbx5; K and L) optic vesicle markers was also undetectable in Six3-mutant embryos. (M-P) At this stage, expression of Mitf remained in the mutant optic vesicles. Scale bar: $100 \mu \mathrm{m}$.

ized in the developing optic vesicles (Figure 2C); however, in the mutant embryos its expression was missing in the most dorsal part of the optic vesicles (Figure 2D) and was abnormally present in the midline region of the forebrain (Figure 2D).

A Six3-related family member, Six6, is expressed in the presumptive ventral optic stalk, the ventral portion of the future NR, and later in the NR, optic chiasm, and optic stalk (9). Six6-null embryos exhibit variable degrees of retinal hypoplasia associated with the absence of the optic chiasm and optic nerve (19). We detected strong expression of Six 6 in the optic vesicles and midline part of the ventral forebrain in E9.0 control embryos (Figure 2, E and G). This expression was undetectable in Six 3 conditional-mutant littermates (Figure 2, F and H).

Similarly, the expression of the presumptive ventral NR marker Vax2 (10) and that of the presumptive dorsal NR marker Tbx5 (11) was also missing in the mutant embryos (Figure 2, I-L). Instead, at this stage, expression of the presumptive RPE marker Mitf (12) remained in the mutant optic vesicles (Figure 2, M-P).
The transcription factor Sox2 is expressed in neural and placodal primordia at E8.5 (31), and its activity is required to regulate the competence of NR progenitors; $10 \%$ of humans with anophthalmia or severe microphthalmia are haploid insufficient for Sox2 (32). We observed no obvious changes in the expression of Sox 2 in the anterior neural region, including the optic vesicle territory, in Six 3 conditional-mutant embryos (Supplemental Figure 2).

Together, these results argue that the conditional deletion of Six 3 has no major consequences on the initial process of optic vesicle formation; instead, it arrested the subsequent process of NR specification, as indicated by the lack of expression of some important players in the mutant optic vesicles as early as E9.0.

To determine whether the observed defects were caused by alterations in cell proliferation or cell death, we performed pH3 immunostaining, TUNEL assays, and activated caspase-3 immunostaining in E8.5-E9.0 embryos. No significant difference was observed in the number of $\mathrm{pH} 3$-positive cells ( $P=0.525, t$ test) 

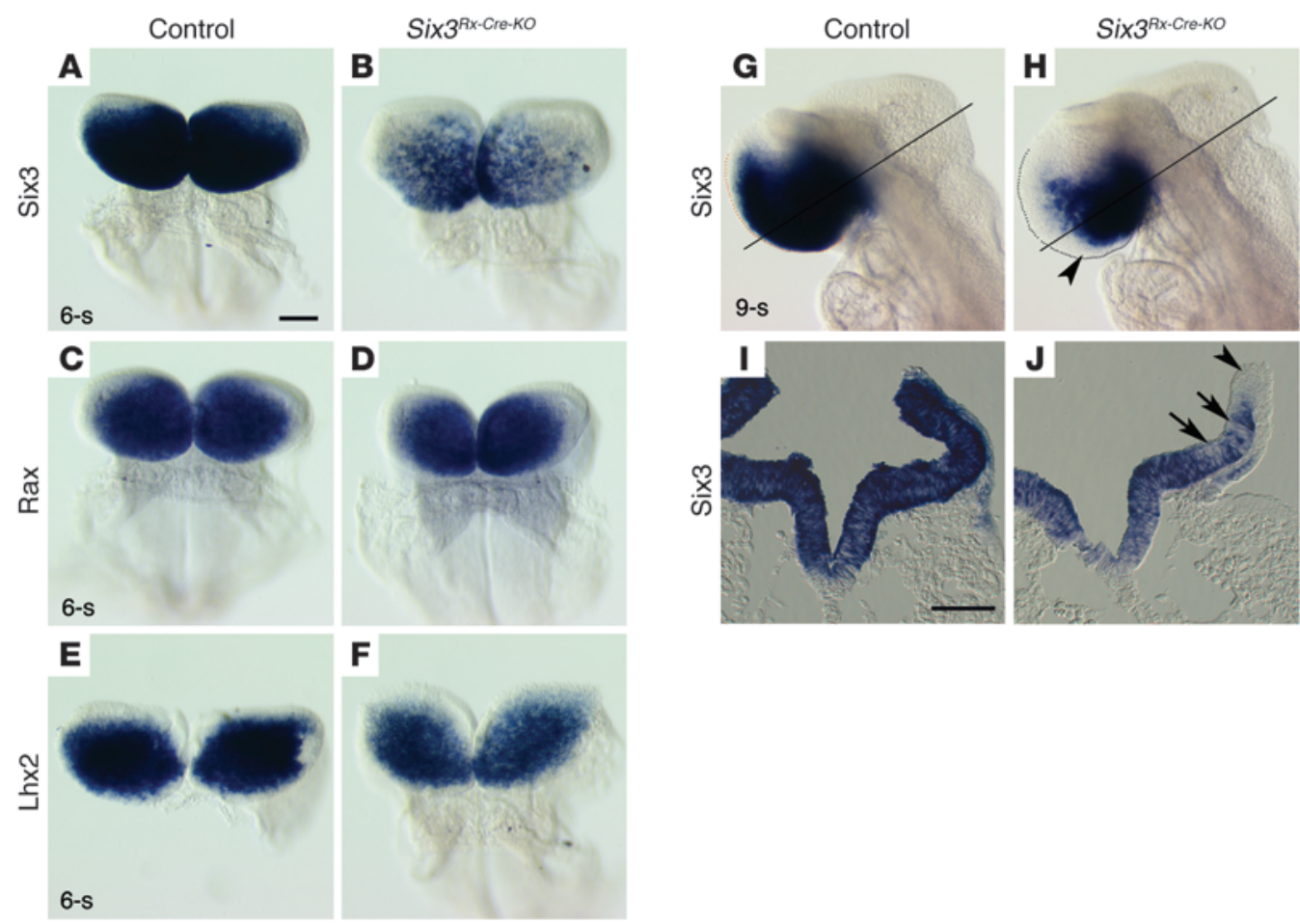

\section{Figure 3}

Eye field is induced in Six ${ }^{R x-C r e-K O}$ embryos. Expression of typical eye field markers such as Six3 (A), Rax (C), and Lhx2 (E) can be detected by whole-mount in situ hybridization analysis of 6-somite staged wild-type embryos. Although reduced, the expression of these markers persists in the eye field territory of Six3 conditional-mutant littermates (B, D, and F). At the 9-somite stage, Six3 expression includes the evaginating optic vesicles ( $\mathbf{G}$ and $\mathbf{I})$. In conditional-mutant littermates, Six3 expression was reduced in a mosaic manner in most of that region (arrows in $\mathbf{J}$ ) and in the rostral margin expression was almost completely absent (arrowheads in $\mathbf{H}$ and $\mathbf{J}$ ). Scale bar: $100 \mu \mathrm{m}$.

between control embryos $(13.2 \% \pm 1.5 \%)$ and Six 3 conditionalmutant embryos $(11.3 \% \pm 2.0 \%)$. (Supplemental Figure 3, C and D). At this stage, $\mathrm{TUNEL}^{+}$cells or activated caspase- $3^{+}$cells were observed in the ventral part of the optic vesicles of control embryos (Supplemental Figure 3, E and G); these cells were absent in the Six3-deficient littermates (Supplemental Figure 3, F and H), and no ectopic apoptosis was observed. Only at later stages (16-somite stage) did we observe some limited ectopic apoptosis toward the dorsal part of the mutant optic vesicles of Six3-deficient embryos (Supplemental Figure 3J). Thus, it is unlikely that drastic alterations in proliferation or apoptosis are responsible for the NR defects observed in early stage Six3-mutant embryos.

Eye field is induced in Six 3 conditional-mutant embryos. Six3 is normally expressed in the mouse eye field territory, the region of the anterior neural plate from which the eyes eventually form at E8.5 (23). Analysis of X-gal-stained $R x$-Cre;R26R embryos identified the presence of $\mathrm{Rx}-\mathrm{Cre}^{+}$cells in the anterior neural plate territory at E7.5 (Supplemental Figure 1). As development progresses, X-gal ${ }^{+}$ cells were also observed in the eye field territory and developing optic vesicles (Supplemental Figure 1).

To determine whether the lack of NR observed in Six $3^{\text {Rx-Cre-KO }}$ embryos could be the consequence of early defects in eye field specification or formation, we analyzed the expression of Six 3 and that of the eye field markers Rax (14) and $L b x 2$ (8) at E8.5. Six3 started to be deleted in a mosaic manner as early as the 6 -somite stage (Figure 3B). However, expression of the eye field markers Rax (14) and Lhx2 (8) was largely unaffected (Figure 3, D and F). Mosaic deletion of Six3 was also evident at the 9-somite stage; however, at that stage, a more efficient deletion was observed toward the rostral margin of the neural ridge (Figure 3, $\mathrm{H}$ and J).

In summary, deletion of Six 3 from the anterior neural plate and eye field of Six $3^{R x-C r-K O}$ embryos did not obviously affect the process of eye field induction. Importantly, this early mosaic deletion was sufficient to alter the levels of Six 3 expression in the rostral margin of the anterior neural ridge and the region of the optic evaginations at the 9-somite stage (Figure 3, G-J). Furthermore, we observed some of the first obvious morphologic defects at this stage in the optic vesicles territory that failed to evaginate properly (Figure 3J).

Rostral expansion of $\mathrm{Wnt} 8 \mathrm{~b} / \beta$-catenin signaling is an early response to Six 3 deletion. As indicated above, the earliest alterations in Six $3^{R x-C r e-K O}$ embryos were detected at around the 6- to 9-somite stage, when Six 3 expression in the eye field territory and anterior neural ridge was reduced. Therefore, to gain insight into the molecular mechanisms that lead to the subsequent NR specification defects, we performed microarray analysis of dissected anterior neural plate territory from 8- to 9-somite staged Six $3^{R x-C r e-K O}$ embryos and control littermates. Among the genes that exhibited significant expression change were several components of the Wnt signaling pathway including Wnt8b (data not shown).

Work performed in zebrafish has suggested that $\mathrm{Wnt} / \beta$-catenin signaling antagonizes eye field specification through the activity of wnt8b and Fz8a (27). In mouse embryos at the 8-somite stage (eye field stage), Wnt $8 b$ expression was localized in the dorsal midline of the caudal forebrain (Figure 4, A and C), at the boundary with the mid- 

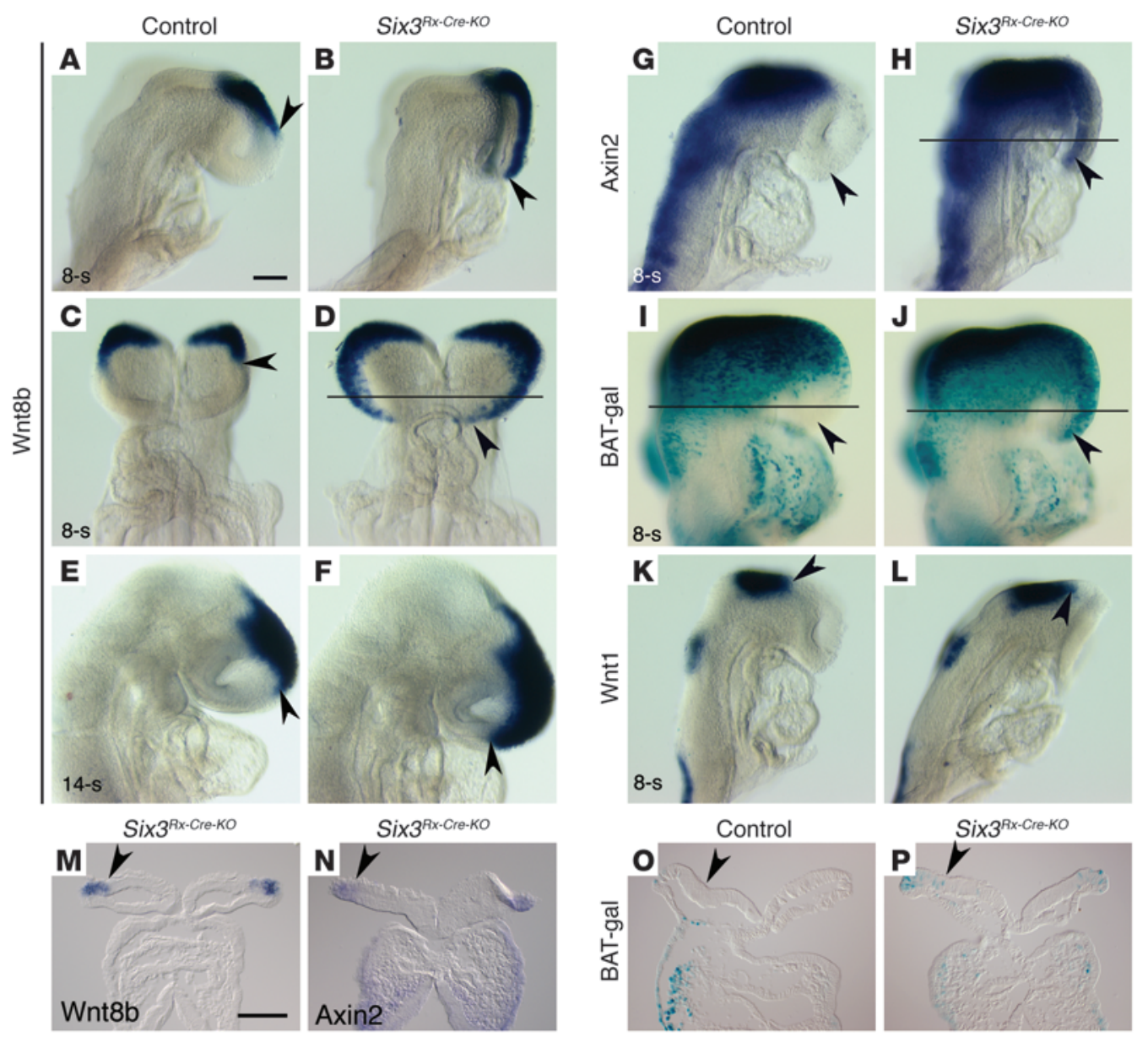

Figure 4

Wnt8b but not Wnt1 expression is rostrally expanded in Six3 ${ }^{\text {Rx-Cre-KO }}$ embryos at E8.5. (A and C) Whole-mount in situ hybridization of 8-somite staged control embryos shows that at this stage, Wnt8b expression is localized at the boundary between the caudal forebrain and midbrain, and its anterior-most boundary (arrowheads) clearly excludes the eye field territory. Wnt8b expression expanded anteriorly (arrowheads in B and $\mathbf{D}$ ) into the developing telencephalon and eye field territory of Six3 conditional-mutant littermates. This ectopic expansion remains in laterstaged mutant embryos (E and $\mathbf{F})$. Accordingly, expression of the Wnt/ $\beta$-catenin signaling readouts, Axin2 $(\mathbf{G}$ and $\mathbf{H})$, and the BAT-gal reporter (I and $\mathbf{J}$ ), were also anteriorly expanded (arrowheads). The expression of Wnt1 was unaffected in Six3-mutant embryos (arrowheads in K and $\mathbf{L})$. Transverse sections at the level of the optic pit indicated by the dash in the embryos shown in $\mathbf{D}, \mathbf{H}, \mathbf{I}$, and $\mathbf{J}$ are shown in $\mathbf{M}, \mathbf{N}, \mathbf{O}$, and $\mathbf{P}$, respectively. Scale bar: $100 \mu \mathrm{m}$.

brain (33), and did not overlap with that of Six3, which was located in the anterior neural plate at this stage (23). Instead, in Six3 conditional-mutant embryos and starting at around this stage, Wnt $8 b$ expression was ectopically expanded into the anterior-most part of the forebrain including the optic pits (Figure 4, B, D, and M). Consistent with this ectopic expansion of Wnt8b, expression of Axin2, an endogenous readout of Wnt/ß-catenin signaling (34), was also anteriorly expanded in the mutant embryos (Figure 4, G, H, and N). Confirming this result further, we found that the activity of the $\mathrm{Wnt} / \beta$-catenin reporter (35) was also rostrally expanded at this stage (Figure 4, I, J, O, and P). This ectopic anterior expansion of the $W n t 8 b$ expression domain was maintained during later developmental stages (Figure 4, E and F).

We previously reported that the standard functional inactivation of Six 3 leads to the rostral expansion of Wnt 1 expression and truncation of the mutant prosencephalon (24). Interestingly, in Six $3^{R x-C r e-K O}$ embryos, Wnt8b expression expanded rostrally but that of Wnt1 was normal (Figure 4, K and L). In fish, Wnt11, Fz5, $F z 8 a$, and $S f r p 1$, in addition to Wnt8b, are also involved in early eye development $(27,36-38)$. In Six $3^{R x-C r-K O}$ embryos, Fzd5 expression was significantly reduced in the anterior neural plate at the 6- to 8 -somite stage (data not shown), but that of Sfrp1 and Fzd8 was unaffected, and Wnt11 was not expressed in the anterior neural plate of control or mutant embryos (data not shown).

Together, these results indicated that functional inactivation of Six 3 in the anterior neural plate (including the eye field territory) led to the specific rostral expansion of Wnt8b expression starting as early as E8.5.

Ectopic Wnt8b expression is sufficient to suppress NR specification in transgenic mouse embryos. Considering that the observed rostral expansion of Wnt8b expression is an early response to Six 3 deficiency and that in zebrafish $w n t 8 b$ antagonizes eye field specification (27), we decided to determine whether ectopic Wnt8b expansion is responsible for the lack of NR specification observed in Six 3 conditional-mutant embryos.

To do this, we generated transient transgenic mouse embryos in which ectopic Wnt8b expression was driven into the anterior forebrain territory by a Fezf2 promoter/regulatory element (39). In 

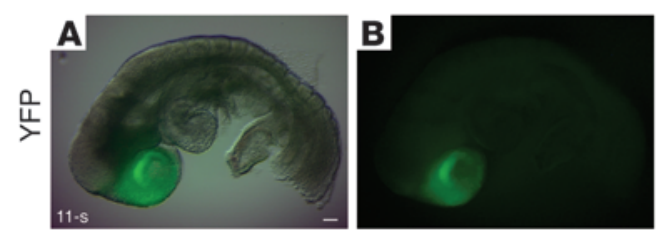

WT
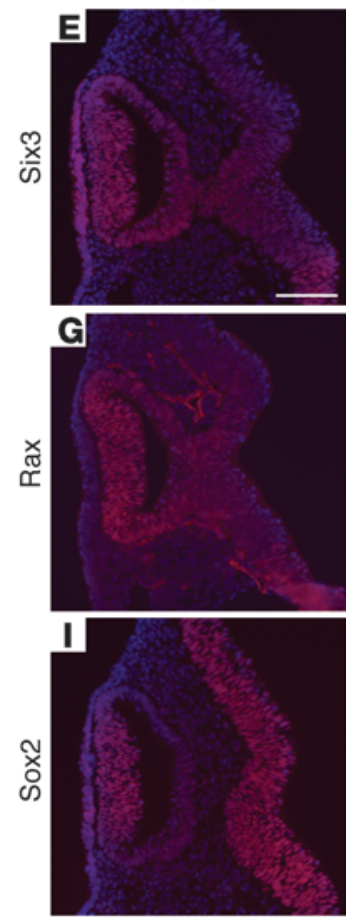

Fez::Wnt8b IRES YFP

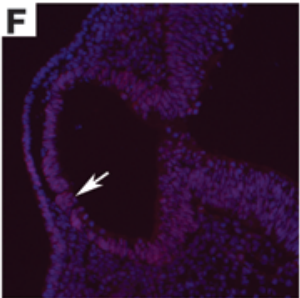

H
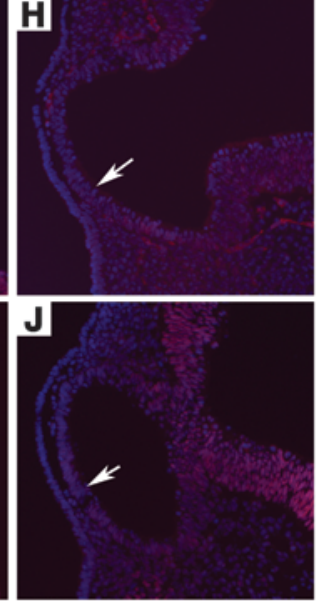
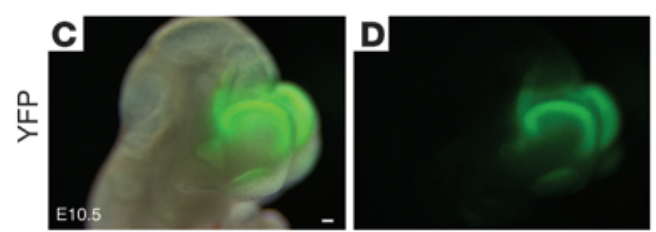

WT

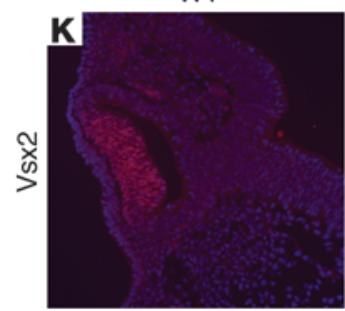

Fez::Wnt8b IRES YFP
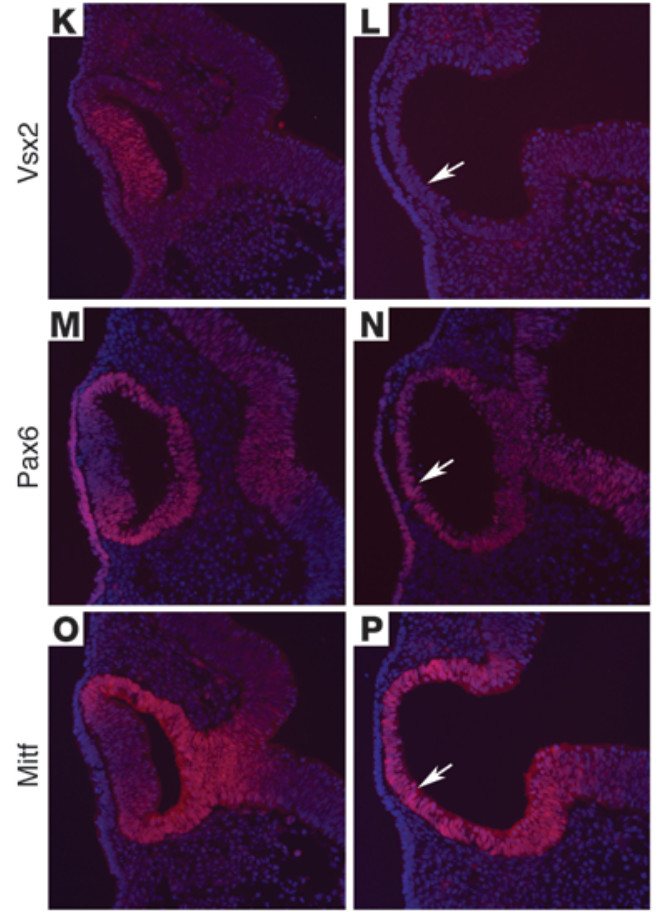

Figure 5

Ectopic Wnt8b expression in transient transgenic mouse embryos recapitulates the retinal phenotype of Six3-deficient embryos. Ectopic Wnt8b expression was driven by a Fezf2 promoter/regulatory element, and transient transgenic embryos were analyzed at E10.5. (A-D) Transgene expression in the forebrain and optic vesicles can be monitored by YFP immunofluorescence starting at the 8- to 11-somite stage. At around E10.5, NR progenitors express Six3 (E), Rax (G), Sox2 (I), and Vsx2 (K). Similarly to Six3 conditional mutants, transgenic embryos ectopically expressing Wnt8b in the rostral forebrain fail to express any of these NR markers (arrows in $\mathbf{F}, \mathbf{H}, \mathbf{J}$, and $\mathbf{L}$ ). In contrast to control embryos (M and $\mathbf{O}$ ) and also similar to Six3 conditional-mutant embryos, the entire transgenic optic vesicle expresses Pax6 (N) and Mitf (P). Scale bars: $100 \mu \mathrm{m}$.

this construct, ectopic transgene expression can be monitored by yellow fluorescent protein (YFP) fluorescence. Although variable, in some of the transgenic embryos, YFP was detected in the forebrain territory as early as the 8- to 11-somite stage (E8.5; Figure 5, $\mathrm{A}$ and $\mathrm{B}$ ) and remained at least until the E10.5 stage (Figure 5, C and D). Analysis of the transgenic embryos at E10.5 revealed that similar to Six $3^{R x-C r e-K O}$ embryos, expression of the NR markers Six3, Rax, Sox2, and Vsx2 was missing or drastically reduced (Figure 5, $\mathrm{E}-\mathrm{L}$ ); in contrast, the entire optic vesicle expressed the RPE-specific marker Mitf (Figure 5P) and Pax6, a gene that at this stage is expressed in the NR and RPE (Figure 5, M and N).

In summary, these results demonstrated that ectopic expression of Wnt8b in the early mouse forebrain was sufficient to arrest NR specification and recapitulated the phenotype observed in Six 3 conditional-deficient embryos at E10.5.

Six3 is an in vivo transcriptional regulator of Wnt8b. One possible explanation for the ectopic anterior expansion of Wnt8b is that Six 3 activity directly maintains a Wnt8b-free territory at the time and in the region where NR specification normally occurs. Therefore, to determine whether Six3 acts as a direct in vivo repressor of Wnt8b, we performed a detailed bioinformatic analysis of the Wnt $8 b$ genomic locus. We identified 2 highly conserved noncoding regions among human, chicken, zebrafish, and fugu fish: 1 region corresponded to the promoter region, and the other was located in the 3' flanking region (Figure 6A). Visual examination of these 2 regions also revealed several Six3 DNA-binding motifs (ref. 40 and Supplemental Figure 4).

To determine whether Six3 functions as an in vivo regulator of Wnt8b activity, we first performed EMSA that showed that Six3 specifically binds the 2 conserved DNA fragments present in the Wnt8b locus in vitro (Figure 6B). We also determined that forced expression of Six 3 in the presumptive forebrain territory of transgenic mouse embryos (driven by the same Fezf2 promoter/regulatory element; ref. 39 described above) drastically reduced Wnt8b expression in this region (Figure 6, C-E). Finally, ChIP analysis of chromatin isolated from the head and trunk of E8.5 embryos by using a rabbit polyclonal antibody specific for Six3 or normal rabbit IgG was performed and specific regions of the immunoprecipitated chromatin were quantified by quantitative PCR (qPCR). Enrichment was measured as the ratio between 
A

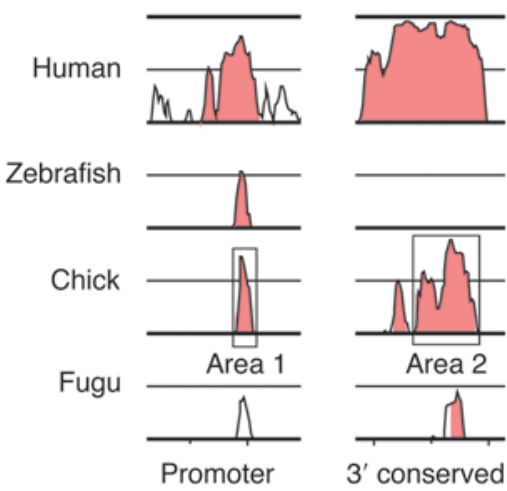

B
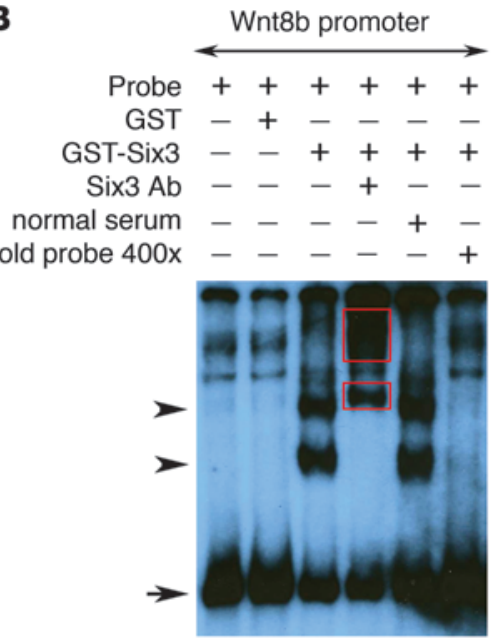
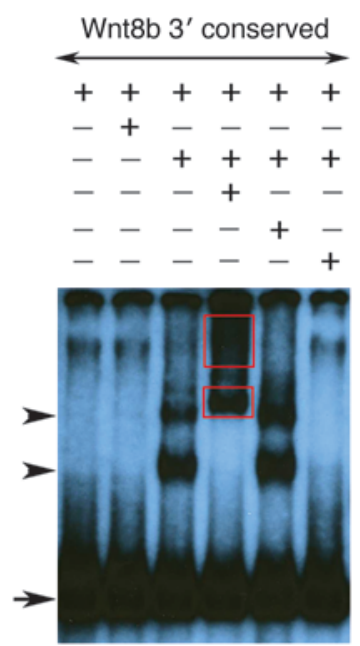

WT

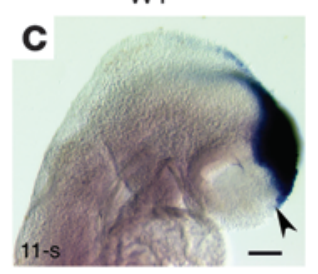

F

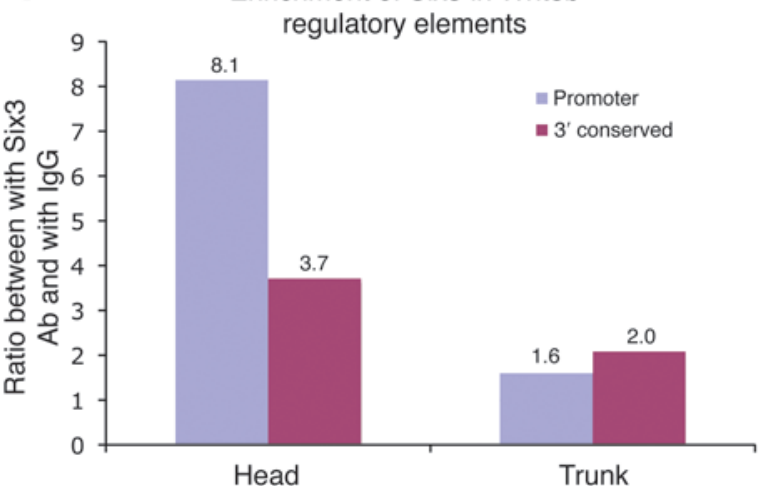

Fez::Six3 IRES YFP

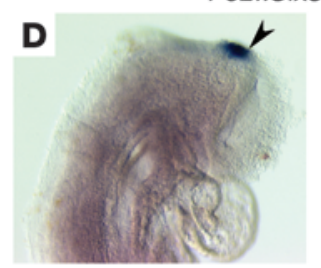

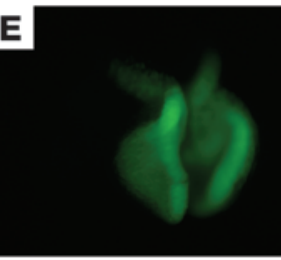

G

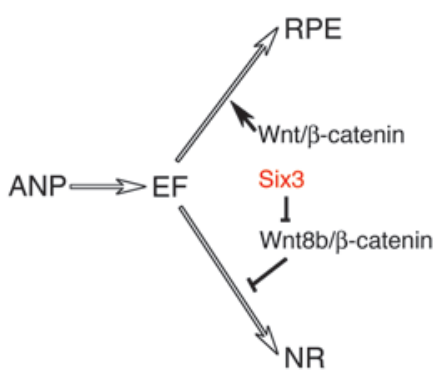

\section{Figure 6}

Six3 binds to Wnt8b regulatory elements in vivo. (A) DNA sequence comparison of the Wnt8b genomic loci of mouse, human, zebrafish, chick, and fugu fish revealed 2 conserved regions located outside the exons: the promoter and a 3 ' flanking fragment. (B) DNA fragments containing potential Six3-binding motifs for both areas were subjected to EMSA analysis. Addition of GST-Six3 protein caused a band shift of the radiolabeled probes, and addition of the Six 3 antibody supershifted (red rectangle) those bands. Cold probes (in $\times 400$ excess) were used to compete with the band shift. (C-E) Forced Six3 expression in the presumptive forebrain territory of E8.5 mouse embryos repressed Wnt8b expression. The anterior boundaries of Wnt8b expression in control $(\mathbf{C})$ and in the transgenic embryo (D) are indicated by arrowheads. Expression of the transgene is monitored by YFP fluorescence (E). (F) Chromatin prepared from the head (Six3 positive) or trunk (Six3 negative) of 8- to 15-somite staged wild-type embryos was used for ChIP using either Six3 antibody or normal rabbit IgG and quantified by SYBR qPCR. The $y$ axis indicates the value ratio between the Six3 and the corresponding IgG immunoprecipitated samples. Six3 was highly enriched in the promoter area and moderately enriched in the 3' flanking area. (G) Working model of NR specification. Normally, the eye field (EF) territory will be derived from the anterior neural plate (ANP). Later on, the NR and RPE will be derived from this region, but while RPE specification requires active Wnt/ $\beta$-catenin signaling, the specification of the NR fate requires its repression by Six3. Scale bar: $100 \mu \mathrm{m}$.

Six3 immunoprecipitated chromatin and the corresponding IgG immunoprecipitated chromatin. Using this approach, we detected an 8.1-fold enrichment of the promoter region and a 3.7-fold enrichment of the $3^{\prime}$ flanking region (Figure 6F).

Together these results argue that Six3 directly suppresses $W n t 8 b$ expression in vivo in the anterior neural plate territory of early mouse embryos.

\section{Discussion}

In vertebrates, eye formation starts with the regionalization and specification of the eye field in the anterior neural plate. Within this region, retinal specification is initiated upon the formation of the optic sulci, the 2 lateral grooves of the anterior neuroectoderm from which the optic vesicles eventually evaginate. The transcription factors Rax, Six3, Six 6, Lhx2, and Pax6 regulate eye development and are 
coexpressed during most of the steps leading to NR differentiation $(7,9,14,19,23)$. Gain-of-function approaches to increase the expression of most of these genes in fish, frog, and mouse embryos are sufficient to promote the formation of retinal tissue (3, 14, 41-44). In contrast, null mutations in some of these same genes (e.g., Rax, Pax6, and $L h x 2)$ arrest development at various key steps during eye formation $(14,15,45)$. In addition, $V s x 2$ activity is known to be required for maintaining NR identity $(20,21)$, and Wnt/ $\beta$-catenin signaling is required for RPE development $(46,47)$. Despite this knowledge, until now no information was available about the specific genes and mechanisms that guide NR specification.

In this article, we demonstrate that conditional inactivation of Six 3 in the mouse anterior neural plate including the eye field is sufficient to arrest the specification of the NR but not that of the RPE. We showed that in Six 3 conditional-mutant embryos, the initial step of eye field induction is not affected; however, later on, the specification of the NR is arrested, as revealed by the lack of expression of the presumptive NR markers Six6, Vax2, Tbx5, and Vsx2 in the mutant optic vesicles. We determined that the ectopic rostral expansion of Wnt8b expression as early as E8.5 is a key early response to Six 3 functional inactivation. Thus, we conclude that the specification of the NR and RPE fates from their common eye field progenitors is under the control of distinct molecular programs and that Six 3 is specifically required for NR specification.

Regionalization of the vertebrate forebrain requires the inhibition of posteriorizing Wnt signaling $(24,48)$, and we have previously shown that Six3 suppression of Wnt1 activity in the anterior neuroectoderm is essential for vertebrate telencephalic development (24). Similarly, the antagonism of the Wnt/ $\beta$-catenin pathway is also necessary during zebrafish eye field development because its activity suppresses eye field formation $(27,37)$. However, this work did not identify the mechanisms by which Wnt activity was specifically antagonized, nor was this activity directly linked to the process of NR specification. We have now identified Wnt $8 b$ as another direct target of Six 3 and demonstrated that Six 3 suppression of $W n t 8 b$ expression in the anterior neural plate is required during NR specification in mammalian embryos. Our data showing that NR specification was specifically suppressed upon the ectopic anterior expansion of Wnt8b in Six 3 conditional-mutant embryos or after forced expression of Wnt8b in transient transgenic embryos conclusively demonstrated that Wnt $8 b$ expression suppresses NR specification during the formation of the mammalian eye and that Six 3 is the direct in vivo antagonist of this Wnt signaling activity during this process.

Currently, we do not know which other gene or genes involved in early eye development are affected by the conditional inactivation of Six 3 or by the ectopic rostral expansion of Wnt8b. Among published reports describing a series of key transcription factors that regulate early eye development, Six 3 is the first gene to be described whose functional inactivation results in the arrest of neuroretinal specification in mammals. The fact that the described eye phenotypes of Six6-, Vax2-, Fzd5-, or Tbx5-mutant embryos differ from that of Six 3 mutants suggests that these genes probably do not contribute to the neuroretinal-specification phenotype observed in Six 3 conditional-mutant embryos. However, it is reasonable to speculate that some of these genes indirectly contribute downstream of Six 3 to regulate later aspects of retinal morphogenesis. In fact, we determined that normal $F z d 5$ expression is absent in the optic vesicles of E9.5 Fez:Wnt8b transgenic embryos (data not shown). This result suggests that $F z d 5$ is a direct target affected by the ectopic rostral expansion of Wnt8b activity, and its downregulation in Six 3 conditional mutants could directly or indirectly affect the morphogenesis of the optic cups.

Interestingly, although the ectopic rostral expansion of Wnt8b was sufficient to suppress NR specification in Six3 conditionalmutant embryos or in transient transgenic embryos, the specification of the RPE was unaffected. This result suggests that the specification of these 2 cell types (NR and RPE) is under the control of distinct molecular programs and that $\mathrm{Six} 3$ is specifically required for the one leading to NR specification. Related to this idea, it was recently reported that $\mathrm{Wnt} / \beta$-catenin signaling is essential for RPE specification $(46,47)$. Thus, although in zebrafish, canonical Wnt/ $\beta$-catenin signaling antagonizes eye field specification and noncanonical Wnt signaling promotes early eye development (27), our work in mammals indicates that proper balancing of Wnt/ $\beta$-catenin signaling is essential to drive common eye field retinal progenitors to differentiate into either NR or RPE progenitors. In this case, Six 3 activity is necessary to maintain a Wnt8b-free territory in the rostro-ventral margin of the anterior neural plate so that NR specification can normally occur.

Regionalization of the vertebrate forebrain requires that Wnt/ $\beta$-catenin signaling is gradually suppressed from the anterior neuroectoderm. Our work has now revealed that mammalian NR specification also requires a regulated balance of the activity of $W n t / \beta$-catenin signaling in the developing eye field. We can conclude that Six3-mediated suppression of various members of the Wnt signaling pathway is a time-dependent, necessary step during the development of the mammalian forebrain and NR. Our results also suggest that fine tuning the timing of Six 3 suppression is essential in determining which Wnt family member will be antagonized and therefore in determining the telencephalic and NR fates in the anterior neural plate. Although telencephalic formation requires Six3-mediated suppression of Wnt1 at the 0 - to 6-somite stage, NR specification requires Six3-mediated suppression of Wnt8b a few hours later at the 6-to 8-somite stage. The knowledge gained from this study may help future efforts aimed toward controlling the differentiation of retinal cells in vivo and in vitro in embryonic stem cells or induced pluripotent stem cells.

\section{Methods}

Mice. The Six $3^{F / F}$ and Six $3^{+/ \Delta}$ (25), Rx-Cre (26), BAT-gal (ref. 35; Jackson Laboratories), and $R 26 R$ (Jackson Laboratories) mouse strains were maintained in the NMRI background and genotyped as previously reported. To generate Fez-Wnt8b IRES YFP transgenic embryos, the Fez-Wnt8b IRES YFP cassette was excised from the plasmid by digestion with SalI; purified DNA was injected into mouse zygotes using standard protocols; and embryos were harvested at specific stages. Transgenic embryos were identified by YFP fluorescence under the dissecting stereomicroscope, and the genotype was confirmed by PCR for YFP. Animal experiments were approved by the Animal Care and Use Committee of St. Jude Children's Research Hospital.

Plasmids. The plasmid Fez:Wnt8b IRES YFP for ectopic Wnt8b expression in the mouse forebrain was constructed by fusing a $2.8-\mathrm{kb}$ section of the mouse Fezf2 promoter/regulatory element (39), a 1-kb cDNA fragment containing the entire mouse Wnt8b coding sequence generated by PCR from a Wnt8b cDNA plasmid (clone ID 40056929; Open Biosystems), and an IRES-Venus-SV40 polyA cassette.

The plasmid Fez:Six 3 IRES YFP for ectopic Six 3 expression in the mouse forebrain was constructed by replacing Wnt8b by the Six 3 coding sequence in Fez:Wnt8b IRES YFP. 
Immunohistochemistry and $m R N A$ in situ bybridization. Standard protocols (25) were used. The following antibodies were used: anti-Six3 (1:400; provided by G. Oliver), anti-Rax (1:1000; Riken/CDB), anti-Sox2 (1:800; Chemicon), anti-Mitf (1:2,000; provided by H. Arnheiter, National Institute of Neurological Disorders and Stroke, NIH, Bethesda, Maryland, USA), anti-Pax6 (1:500; Covance), anti-Vsx2 (1:200; Abcam), anti-pH3 (1:2000; Upstate), anti-active caspase-3 (1:200, antigen retrieval, and TSA amplification; BD Biosciences). The following plasmids were used to generate probes for in situ hybridization: Six 3 (XbaI/T7; provided by G. Oliver), Rax (HindIII/T7; provided by P. Mathers, West Virginia University School of Medicine, Morgantown, West Virginia, USA), Wnt8b (XbaI/T3; provided by B. Hogan, Duke University Medical Center, Durham, North Carolina, USA), Axin2 (NotI/T3; provided by F. Costantini, College of Physicians and Surgeons, Columbia University, New York, New York, USA), Wnt1 (EcoRI/ SP6; provided by M. Torres, Centro Nacional de Investigaciones Cardiovasculares, Madrid, Spain), Six6 (XbaI/T7; provided by X. Li, Children's Hospital, Boston, Massachusetts, USA), Fzd5 (NotI/T7; IMAGE 445088), Fzd8 (NcoI/T3; Open Biosystems 40130820), Wnt11 (XhoI/T3; provided by A. McMahon, Harvard University, Cambridge, Massachusetts, USA), Vax2 (NcoI/T7; provided by C. Cepko, Harvard University, Boston, Massachusetts, USA), Tbx5 (SpeI/T7; provided by A. Joyner, Skirball Institute of Biomolecular Medicine, New York University School of Medicine, New York, New York, USA), Mitf (HindIII/T3; provided by H. Arnheiter), Sox2 (Xhol/T3; provided by L. Robertson, Sir William Dunn School of Pathology, University of Oxford, Oxford, United Kingdom), Lhx2 (NotI/T7; provided by $\mathrm{H}$. Westphal, National Institute of Child Health and Human Development, NIH, Bethesda, Maryland, USA).

TUNEL and proliferation assays. Three 8- to 9-somite control and 3 conditional Six 3 mutant embryos were sectioned (coronal) through the optic vesicles region, and 6 sections of each were immunostained for $\mathrm{pH} 3$ and counterstained for DAPI. Their respective numbers were counted using Adobe Photoshop software. The percentage of $\mathrm{pH} 3$-positive cells was calculated and the $\mathrm{pH} 3$ index was expressed as mean $\pm \mathrm{SD}$. Statistical significance was analyzed by paired 2 -tailed $t$ test.

TUNEL assay was performed using ApopTag kit (Millipore) according to the manufacture's instruction. At least 2 embryos from the control or the conditional Six 3 mutant embryos were used.

DNA sequence comparison. Available DNA genomic sequences of mouse, human, chick, zebrafish, and fugu fish Wnt $8 b$ locus, including 5-kb upstream and $5-\mathrm{kb}$ downstream sequences, were downloaded from the Ensembl database and subjected to sequence comparison using the VISTA tool.

EMSA assay. The 2 DNA fragments for EMSA were amplified by PCR using the primers indicated in Supplemental Figure 4 and then subcloned into the T-easy vector (Promega) and sequenced for confirmation. These 2 fragments were released from the plasmid by using NotI and labeled with $[\alpha-32 \mathrm{P}] \mathrm{dCTP}$ using Klenow. Briefly, for EMSA, $1 \mu \mathrm{l}$ of the labeled probes were incubated with $0.5 \mu \mathrm{l}$ of purified GST or GST-Six 3 protein in $15 \mu \mathrm{l}$ of binding buffer (25 mM Hepes, pH 7.5; $100 \mathrm{mM} \mathrm{KCl} ; 1$ mM EDTA; $10 \mathrm{mM}$ $\mathrm{MgCl}_{2} ; 0.1 \% \mathrm{NP}-40 ; 5 \%$ glycerol; and $\left.1 \mathrm{mM} \mathrm{DTT}\right)$ supplemented with $1 \mu \mathrm{l}$ poly $(\mathrm{dI}-\mathrm{dC})(0.6 \mathrm{mg} / \mathrm{ml})$ and $0.5 \mu \mathrm{lBSA}(10 \mathrm{mg} / \mathrm{ml})$ for 20 minutes at room temperature. Rabbit polyclonal antibody specific for Six $3(0.5 \mu \mathrm{l})$ or normal rabbit serum $(0.5 \mu \mathrm{l})$ was added for another 20 minutes, and excess cold probe (400-fold more than hot probe) was added for competition. The DNA protein complex was resolved in $3.5 \%$ nondenaturing protein gel. Electrophoresis was done in $\times 0.5 \mathrm{TBE}$ buffer at $150 \mathrm{~V}$ at room temperature for several hours. The gel was dried, and the protein-DNA complexes were visualized by autoradiography.

ChIP assay and $q$ PCR. Chromatin isolated from the head (Six3 expressing) and trunk (Six3 negative) of E8.5 (8-15 somite stage) wild-type embryos was subjected to a ChIP assay with either Six 3 antibody (our own) or normal IgG, and the precipitate was quantified by SYBR qPCR (Applied Biosystems). The enrichment was expressed as the value ratio between the Six3 immunoprecipitated sample over the corresponding IgG immunoprecipitated sample. The detailed protocol is provided in Supplemental Methods.

\section{Acknowledgments}

We want to thank H. Arnheiter, P. Mathers, B. Hogan, F. Constantine, X. Li, A. McMahon, C. Cepko, M. Torres, L. Robertson, H. Westphal, and A. Joyner for probes and antibodies. We also thank B. Sosa-Pineda and members of G. Oliver's laboratory for helpful discussions, X. Geng for critical reading of this manuscript, G. Grosveld and staff members of the St. Jude Transgenic/Gene Knockout Facility for the generation of transgenic embryos, and Angela McArthur for editing of this manuscript. This work was supported in part by NIH grant EYEY12162 to G. Oliver, Cancer Center Support grant CA-21765, and the American Lebanese Syrian Associated Charities (ALSAC).

Received for publication April 2, 2010, and accepted in revised form August 4, 2010.

Address correspondence to: Guillermo Oliver, Department of Genetics and Tumor Cell Biology, St. Jude Children's Research Hospital, 262 Danny Thomas Place, Memphis, Tennessee 38105, USA. Phone: 901.595.2697; Fax: 901.595.6035; E-mail: guillermo. oliver@stjude.org.
1. Klassen H, Sakaguchi DS, Young MJ. Stem cells and retinal repair. Prog Retin Eye Res. 2004;23(2):149-181.

2. Lamba D, Karl M, Reh T. Neural regeneration and cell replacement: a view from the eye. Cell Stem Cell. 2008;2(6):538-549.

3. Chow RL, Lang RA. Early eye development in vertebrates. Annu Rev Cell Dev Biol. 2001;17:255-296.

4. Inoue T, Nakamura S, Osumi N. Fate mapping of the mouse prosencephalic neural plate. Dev Biol. 2000;219(2):373-383

5. Livesey FJ, Cepko CL. Vertebrate neural cell-fate determination: lessons from the retina. Nat Rev Neurosci. 2001;2(2):109-118.

6. Viczian AS, Solessio EC, Lyou Y, Zuber ME. Generation of functional eyes from pluripotent cells. PLoS Biol. 2009;7(8):e1000174.

7. Zuber ME, Gestri G, Viczian AS, Barsacchi G, Harris WA. Specification of the vertebrate eye by a network of eye field transcription factors. Development. 2003;130(21):5155-5167.

8. Tetreault N, Champagne MP, Bernier G. The LIM homeobox transcription factor Lhx2 is required to specify the retina field and synergistically cooperates with Pax6 for Six 6 trans-activation. Dev Biol. 2009;327(2):541-550.

9. Jean D, Bernier G, Gruss P. Six6 (Optx2) is a novel murine Six3-related homeobox gene that demarcates the presumptive pituitary/hypothalamic axis and the ventral optic stalk. Mech Dev. 1999; 84(1-2):31-40.

10. Mui SH, Hindges R, O'Leary DD, Lemke G, Bertuzzi $S$. The homeodomain protein Vax2 patterns the dorsoventral and nasotemporal axes of the eye. Development. 2002;129(3):797-804.

11. Niederreither K, Vermot J, Messaddeq N, Schuhbaur B, Chambon P, Dolle P. Embryonic retinoic acid synthesis is essential for heart morphogenesis in the mouse. Development. 2001;128(7):1019-1031.

12. Nguyen M, Arnheiter H. Signaling and transcriptional regulation in early mammalian eye development: a link between FGF and MITF. Development. 2000;127(16):3581-3591.
13. Bailey TJ, El-Hodiri H, Zhang L, Shah R, Mathers $\mathrm{PH}$, Jamrich M. Regulation of vertebrate eye development by Rx genes. Int J Dev Biol. 2004; 48(8-9):761-770.

14. Mathers PH, Grinberg A, Mahon KA, Jamrich M. The Rx homeobox gene is essential for vertebrate eye development. Nature. 1997;387(6633):603-607.

15. Porter FD, et al. Lhx2, a LIM homeobox gene, is required for eye, forebrain, and definitive erythrocyte development. Development. 1997;124(15):2935-2944.

16. Zhang L, Mathers PH, Jamrich M. Function of $\mathrm{Rx}$, but not Pax6, is essential for the formation of retinal progenitor cells in mice. Genesis. 2000; 28(3-4):135-142.

17. Baumer N, et al. Retinal pigmented epithelium determination requires the redundant activities of $\mathrm{Pax} 2$ and Pax6. Development. 2003;130(13):2903-2915.

18. Burns CJ, et al. Investigation of Frizzled-5 during embryonic neural development in mouse. Dev Dyn. 2008;237(6):1614-1626.

19. Li X, Perissi V, Liu F, Rose DW, Rosenfeld MG. 
Tissue-specific regulation of retinal and pituitary precursor cell proliferation. Science. 2002; 297(5584):1180-1183.

20. Horsford DJ, Nguyen MT, Sellar GC, Kothary R, Arnheiter H, McInnes RR. Chx10 repression of Mitf is required for the maintenance of mammalian neuroretinal identity. Development. 2005;132(1):177-187.

21. Rowan S, Chen CM, Young TL, Fisher DE, Cepko CL. Transdifferentiation of the retina into pigmented cells in ocular retardation mice defines a new function of the homeodomain gene Chx10. Development. 2004;131(20):5139-5152.

22. Hodgkinson CA, et al. Mutations at the mouse microphthalmia locus are associated with defects in a gene encoding a novel basic-helix-loop-helixzipper protein. Cell. 1993;74(2):395-404.

23. Oliver G, Mailhos A, Wehr R, Copeland NG, Jenkins NA, Gruss P. Six3, a murine homologue of the sine oculis gene, demarcates the most anterior border of the developing neural plate and is expressed during eye development. Development. 1995;121(12):4045-4055.

24. Lagutin OV, et al. Six3 repression of Wnt signaling in the anterior neuroectoderm is essential for vertebrate forebrain development. Genes Dev. 2003;17(3):368-379.

25. Liu W, Lagutin OV, Mende M, Streit A, Oliver G. Six3 activation of Pax6 expression is essential for mammalian lens induction and specification. EMBOJ. 2006;25(22):5383-5395.

26. Swindell EC, et al. Rx-Cre, a tool for inactivation of gene expression in the developing retina. Genesis. 2006;44(8):361-363.

27. Cavodeassi F, et al. Early stages of zebrafish eye formation require the coordinated activity of Wnt 11 , Fz5, and the Wnt/beta-catenin pathway. Neuron. 2005;47(1):43-56.

28. Liu C, Nathans J. An essential role for frizzled 5 in mammalian ocular development. Development.
2008;135(21):3567-3576.

29. Van Raay TJ, et al. Frizzled 5 signaling governs the neural potential of progenitors in the developing Xenopus retina. Neuron. 2005;46(1):23-36.

30. Zhang J, Fuhrmann S, Vetter ML. A nonautonomous role for retinal frizzled-5 in regulating hyaloid vitreous vasculature development. Invest $O p h$ thalmol Vis Sci. 2008;49(12):5561-5567.

31. Avilion AA, Nicolis SK, Pevny LH, Perez L, Vivian N, Lovell-Badge R. Multipotent cell lineages in early mouse development depend on SOX2 function. Genes Dev. 2003;17(1):126-140.

32. Taranova OV, et al. SOX2 is a dose-dependent regulator of retinal neural progenitor competence. Genes Dev. 2006;20(9):1187-1202.

33. Richardson M, Redmond D, Watson CJ, Mason JO. Mouse Wnt8B is expressed in the developing forebrain and maps to chromosome 19. Mamm Genome. 1999;10(9):923-925.

34. Jho EH, Zhang T, Domon C, Joo CK, Freund JN, Costantini F. Wnt/beta-catenin/Tcf signaling induces the transcription of Axin2, a negative regulator of the signaling pathway. Mol Cell Biol. 2002;22(4):1172-1183.

35. Maretto S, et al. Mapping Wnt/beta-catenin signaling during mouse development and in colorectal tumors. Proc Natl Acad Sci U S A. 2003;100(6):3299-3304.

36. Esteve P, Lopez-Rios J, Bovolenta P. SFRP1 is required for the proper establishment of the eye field in the medaka fish. Mech Dev. 2004; 121(7-8):687-701.

37. Kim SH, et al. Specification of an anterior neuroectoderm patterning by Frizzled8a-mediated Wnt8b signalling during late gastrulation in zebrafish. Development. 2002;129(19):4443-4455.

38. Kim HS, et al. Eye field requires the function of Sfrp1 as a Wnt antagonist. Neurosci Lett. 2007;414(1):26-29.

39. Hirata T, Nakazawa M, Muraoka O, Nakayama R,
Suda Y, Hibi M. Zinc-finger genes Fez and Fez-like function in the establishment of diencephalon subdivisions. Development. 2006;133(20):3993-4004.

40. Zhu CC, Dyer MA, Uchikawa M, Kondoh H, Lagutin OV, Oliver G. Six3-mediated auto repression and eye development requires its interaction with members of the Groucho-related family of corepressors. Development. 2002;129(12):2835-2849.

41. Bernier G, Panitz F, Zhou X, Hollemann T, Gruss P, Pieler T. Expanded retina territory by midbrain transformation upon overexpression of Six $6(\mathrm{Optx} 2)$ in Xenopus embryos. Mech Dev. 2000;93(1-2):59-69.

42. Lagutin O, Zhu CC, Furuta Y, Rowitch DH, McMahon AP, Oliver G. Six3 promotes the formation of ectopic optic vesicle-like structures in mouse embryos. Dev Dyn. 2001;221(3):342-349.

43. Loosli F, Winkler S, Wittbrodt J. Six3 overexpression initiates the formation of ectopic retina. Genes Dev. 1999;13(6):649-654.

44. Zuber ME, Perron M, Philpott A, Bang A, Harris WA. Giant eyes in Xenopus laevis by overexpression of XOptx2. Cell. 1999;98(3):341-352.

45. Grindley JC, Davidson DR, Hill RE. The role of Pax-6 in eye and nasal development. Development. 1995;121(5):1433-1442.

46. Fujimura N, Taketo MM, Mori M, Korinek V, Kozmik Z. Spatial and temporal regulation of Wnt/beta-catenin signaling is essential for development of the retinal pigment epithelium. Dev Biol. 2009;334(1):31-45.

47. Westenskow P, Piccolo S, Fuhrmann S. Betacatenin controls differentiation of the retinal pigment epithelium in the mouse optic cup by regulating Mitf and Otx2 expression. Development. 2009;136(15):2505-2510.

48. Houart C, Caneparo L, Heisenberg C, Barth K, Take-Uchi M, Wilson S. Establishment of the telencephalon during gastrulation by local antagonism of Wnt signaling. Neuron. 2002;35(2):255-265. 